\title{
Improved systemic and mucosal antibody responses with a CCR10 ligand adjuvant
}

\author{
N Hutnick ${ }^{1 *}$, DJ Myles ${ }^{1}$, A Ginsberg ${ }^{1}$, AS Khan ${ }^{2}$, J Yan $^{2}$, Z Moldoveanu ${ }^{3}$, J Mestecky ${ }^{3}$, PA Marx ${ }^{4}$, M Kutzler ${ }^{5}$, \\ DB Weiner ${ }^{1}$
}

From AIDS Vaccine 2012

Boston, MA, USA. 9-12 September 2012

\section{Background}

The induction of potent mucosal immune responses will be critical for an effective HIV vaccine, However, a major limitation of current vaccine development is the ability to induce mucosal antibodies by a systemic, non-replicating vector. To address this inadequacy, we have hypothesized that encoding instructions for immune cell targeting to the mucosa in the form of MEC, a mucosal chemokine adjuvant delivered as a plasmid can redirect immune responses in vivo. MEC (CCL28) is normally expressed by epithelium in the skin, lungs, and intestines and it functions to attract CCR10 expressing plasmablasts locally.

\section{Methods}

IIndian rhesus macaques were vaccinated using EP delivery with either a pcon SIVmac239 gag, pol, SIVsm unmatched E660 env vaccine delivered IM alone $(n=5)$, with CCL28 (MEC, $n=5$ ) or a plasmid expressed H1 HA Influenza vaccine alone $(n=4)$ or with $\operatorname{MEC}(n=4)$. SIV Vaccinated animals and 6 naïve controls were challenged vaginally twice weekly for four weeks with 500TCID50 SIVsmE660.

\section{Results}

The inclusion of a CCR10 ligand adjuvant enhanced vaginal and serum IgG and IgA titers compared with DNA alone. In Flu vaccinated animals functional HAI antibody titers were significantly elevated and above the 1:40 titer required for protection in humans with just a single dose of H1HA delivered with the MEC adjuvant. Following SIV challenge monkeys vaccinated with a CCR10 adjuvant showed $89 \%$ protection from the establishment of infection compared $40 \%$ with DNA alone with only $16 \%$ of the naïve animals.

\section{Conclusion}

Mucosal and systemic antibody responses were enhanced with the inclusion of a CCR10 ligand adjuvant. Dose sparing was also observed. DNA vaccination alone improved challenge outcome, and this was further enhanced by the inclusion of a CCR10 ligand adjuvant. The inclusion of mucosal homing chemokines represents a novel approach to induce improved mucosal immune responses by non-live systemic immunization of relevance to HIV infection.

\section{Author details}

${ }^{1}$ University of Pennsylvania, Philadelphia, PA, USA. ${ }^{2}$ Inovio Pharmaceuticals, Blue Bell, PA, USA. ${ }^{3}$ University of Alabama, Birminham, AL, USA. ${ }^{4}$ Tulane University, Covington, LA, USA. ${ }^{5}$ Drexel University, Philadelphia, PA, USA.

Published: 13 September 2012

doi:10.1186/1742-4690-9-S2-P203

Cite this article as: Hutnick et al:: Improved systemic and mucosal antibody responses with a CCR10 ligand adjuvant. Retrovirology 2012 9(Suppl 2):P203.

${ }^{1}$ University of Pennsylvania, Philadelphia, PA, USA

Full list of author information is available at the end of the article

C 2012 Hutnick et al; licensee BioMed Central Ltd. This is an Open Access article distributed under the terms of the Creative Commons 